\title{
Developing Maintainable Application-Centric IoT Ecosystems
}

\author{
Michiel Willocx, Ilse Bohé, Jan Vossaert and Vincent Naessens \\ MSEC, imec-DistriNet \\ KU Leuven, Technology Campus Ghent \\ Gebroeders Desmetstraat 1, 9000 Ghent, Belgium \\ firstname.lastname@kuleuven.be
}

\begin{abstract}
The amount of Internet-of-Things (IoT) ecosystems that are rolled out in various domains is still increasing. Despite their ability to optimize business processes and increase well-being in various ways, many companies are currently struggling to design and maintain complex IoT ecosystems in a cost-efficient way. A major reason is the vendor lock-in trap that is often triggered by sensor-centric application development. This paper presents application-centric development as an alternative approach to tackle maintainability problems in IoT ecosystems. The paradigm shift is supported by a layered architecture SMIoT, which guides the design of Smart and Maintainable IoT ecosystems. The key qualities of the proposed architecture are reconfigurability, context-awareness and separation of concerns. Furthermore, the architectural principles are adopted in an Android framework implementation and validated through the design of a fleet management ecosystem.
\end{abstract}

\section{Keywords-Internet-of-Things; Software Architecture}

\section{INTRODUCTION}

Many companies across multiple sectors are currently exploring the opportunities of applying Internet-of-Things (IoT) technologies in their digital transformation process. In the transport sector, smart IoT applications can support tracking and parameter monitoring of sensitive or expensive items. Depending on the specific use case, the temperature, humidity, vibration and location can be parameters of interest. In care environments, IoT ecosystems allow for more accurate and reliable patient monitoring while at the same time being less intrusive. IoT applications connected to sensors and actuators support elderly people to live in their own houses without compromising safety. Examples are fall detection mechanisms and smart emergency buttons. Even though connecting sensors and actuators via digital networks to computers systems brings major advantages, many system integrators are currently struggling to build maintainable and cost-efficient IoT ecosystems.

Today, sensor-centric development is the predominant paradigm when building IoT ecosystems. This implies that sensors and actuators are selected and anchored in a very early design stage. Thereafter, system integrators start to think about the design and realisation of attractive software applications. However, the lifetime of advanced software applications often outreaches the lifetime of IoT sensors due to their limited cost or harsh conditions in which they are deployed. Unfortunately, many IoT integrations offer no or very limited flexibility when sensors need to be replaced, as sensors are selected at the very beginning. For instance, a temperature sensor from one manufacturer can often not be replaced by another - more robust or cheaper - one from another manufacturer without major code changes due to lack of flexibility during system design. Vendor lock-in is often mentioned as one of the fundamental problems in current IoT deployments. In addition, implementation cycles are often expensive due to the lack of high-level sensor integration support towards application developers. Many IoT sensors currently on the market only offer a low level API, confronting application programmers with low-level connectivity and data representation problems. On its turn, this increases the development cycle times. Moreover, the large gap between business logic and assets on the one hand, and the actual IoT device sensing and actuating on the other hand complicates application development.

This paper proposes a paradigm shift from sensorcentric towards application-centric IoT ecosystem design. The SMIoT architecture facilitates the latter, and supports the development of complex and maintainable IoT applications. The architectural guidance allows for dynamic and reconfigurable IoT sensor and actuator integration, and hides low-level implementation details towards application developers. Hence, the latter can focus on business logic without being expert in IoT sensor technology. The proposed architecture is especially useful for software companies focusing on complex IoT applications in a specific domain or sector. A typical example is a software integrator focusing on innovative health environments, or a company building extensible software ecosystems for fleet management. With complex, we mean that the IoT ecosystems can consist of various IoT applications used by different stakeholders in the domain. For instance, in a care environment, applications can be developed for caregivers, elderly people, family, doctors, nurses, government, insurance... Each stakeholder has a partial view on the overall IoT ecosystem. The software may evolve over time, and its lifetime is typically much longer than the lifetime of the sensor technologies that are plugged in. With complex, we also mean that a prototypical development team typically consists of individuals with complementary skills. Some members focus on implement- 
ing business logic; others are experienced in supporting the right software abstractions for IoT sensor and actuator technology. The layering offered by our architecture offers the right approach for development teams with mixed skills.

Contribution. The contribution of this work is twofold. First, this work proposes application-centric IoT development as a key enabler for building complex but at the same time maintainable IoT ecosystems. The approach is supported by the definition of a software architecture called SMIoT that supports the design and implementation of dynamic and context-aware IoT environments. The layered architecture offers appropriate abstractions for mixed development teams by decoupling application-level concepts from the deployed infrastructure and the interaction with the physical world. The architecture focuses on increased flexibility and aims at tackling the vendor lock-in trap. Second, the architectural insights are incorporated in an Android framework and validated through the design of a fleet management ecosystem consisting of various IoT components.

The remainder of this paper is structured as follows. Section III points to related work. Section III gives an overview of the SMIoT architecture, after which the framework support is described in Section IV Section V validates the architectural concepts, and section VI discusses the proposed architecture. This paper ends with conclusions.

\section{RELATED WORK}

Many communication technologies are often combined in complex IoT ecosystems. Their strengths and constraints are evaluated in many papers [1], [2], [3], [4]. Standardization efforts are presented to enable interoperability. One example is oneM2M [5], which aims at establishing a standardized M2M service layer platform for globally applicable and access-independent M2M services. Other standardization efforts focus on specific application domains such as smart homes [6], smart cities [7] and Industrial Internetof-Things (IIoT) [8]. Although these standardization efforts target interoperability between IoT components from different stakeholders, the IoT market is still very fragmented. Hence, the flexibility of IoT ecosystem providers is significantly decreased if they are restricted to IoT components adhering to a specific standard. The SMIoT architecture focuses on internal standardization (i.e. by IoT integrator itself). This enables IoT integrators to tailor the interface to IoT components to meet the specific requirements of their applications. Wrappers for IoT standards can enable interoperability with a wide range of IoT components, while wrappers for proprietary protocols provide the flexibility to integrate non-standardized IoT components.

An alternative approach for managing the heterogeneity in IoT components is relying on a cloud or gateway platform that provides uniform interfaces to the IoT components that are connected to the platform. For instance, Lea et. al. [9] and Demirkan et al. [10] use a cloud-based hub for developing respectively smart city and healthcare applications. A local gateway setup is used by Yang et. al in their MicroPnP [11] platform. It is a generic zeroconfiguration, plug-and-play wireless sensor platform consisting of a gateway that interacts with sensor nodes on which sensors/actuators can be added without requiring any additional configuration. Access to the sensors is provided via a REST interface on the gateway. A setup with a combination of a local gateway and cloud hub is used by Soliman et al. [12] and Desai et al. [13] in the smart home application domain, and is also adopted by many commercial organizations. For instance, Google 11 Apple 2 and Samsung 3 provide their own smart home IoT platform. Each platform defines APIs that can be supported by thirdparty IoT device developers to enable interoperability. Using a cloud or gateway platform to bootstrap access to IoT devices significantly simplifies management and application development. However, typically multiple applications can be developed in the context of an IoT ecosystems. Each of these applications can have different requirements with respect to privacy, real-time constraints, access control etc. To fulfill advanced requirements, often a hybrid distributed setup that combines both direct sensor-application interactions as well as interactions mediated via gateways or a cloud platform is required [14]. The architecture presented in this paper facilitates application development in the scope of these complex hybrid and distributed setups.

The domain of context-aware services [15], [16] and IoT ecosystems are closely related [17]. Sensor data gathered from IoT devices can be used to establish the context of a user and, subsequently, tailor [18] the services and content provided by the application. Several papers [15], [19] focus on defining the concept of context and describing an ontology [20] to share context between devices. Other research [21], [22] uses context to control access to remote services. For instance, users may only be granted access to highly sensitive corporate documents if they are at the office. The architecture presented in this paper is complementary with the research around context-aware services. Currently context information is often restricted to sensor information obtained from the (mobile) device on which the application is running. Several frameworks [23], [24] have been proposed that enable developers to gather and manage context information using the device's sensors. The SMIoT architecture facilitates access to external IoT sensors, enabling applications to establish the user context beyond the regular sensors available on the device. It also contains a context-aware module to determine which IoT components need to be loaded in the application.

\footnotetext{
${ }^{1}$ https://developers.nest.com

${ }^{2}$ https://www.apple.com/us/shop/accessories/all-accessories/homekit

${ }^{3}$ https://www.smartthings.com/
} 


\section{ARChitecture}

This section gives an overview of the SMIoT architecture. It supports complex and maintainable IoT integrations, and aims at meeting the advanced flexibility and reconfigurability requirements of both system integrators and customers. The architecture facilitates low-cost modifications of IoT ecosystems over time, as new sensor and actuator technologies can be plugged in while keeping the development cost under control. Hence, IoT ecosystems can evolve over time and vendor lock-in can be avoided which, on their turn, can be competitive differentiators for IoT integrators. An overview of the layered architecture and concepts at each layer is given after the discussion of the requirements:

- Intuitive interfaces. Developers want to rely on interfaces that make abstraction of details of the underlying infrastructure. In many cases, they even do not want to be confronted with any IoT sensors or actuators at all. This means that application developers want to invoke methods on assets (such as patients, rooms, cars...) instead of sensors (such as fall detector, light source, gps sensor).

- Separation of concerns. The architecture facilitates development teams consisting of members with complementary skills. Some developers focus on IoT device integration while others provide domain-specific intuitive interfaces to application developers. Finally, application developers focus on realising application logic and do not want to be confronted with the underlying IoT infrastructure (like the sensor model or vendor, communication technology...).

- Reconfigurability. IoT devices can be replaced by other ones - possibly provided by other sensor manufacturers - without affecting the application. Supporting cost-efficient sensor replacement is essential to tackle vendor lock-in, and enables the use of more accurate or less expensive sensors over time, depending on the specific application needs. For example, replacing a Bluetooth temperature sensor by an alternative one that pushes its data directly to the cloud should be possible with limited implementation effort. The application logic remains unchanged.

- Context-awareness. The optimal IoT configuration is loaded and initialized based on contextual parameters, and context may evolve over time. The application can rely on sensor data (like beacon technology and GPS data), user information and external data like time to determine the current context. For instance, while a caregiver can visit multiple patients, the application only needs to access and show the sensor devices of the patient he is visiting.

\section{A. Abstraction Layers}

The SMIoT architecture consists of four abstraction layers: the IoT infrastructure layer, the virtual IoT device layer, the component layer and the application layer. The layers are depicted in Figure 1.

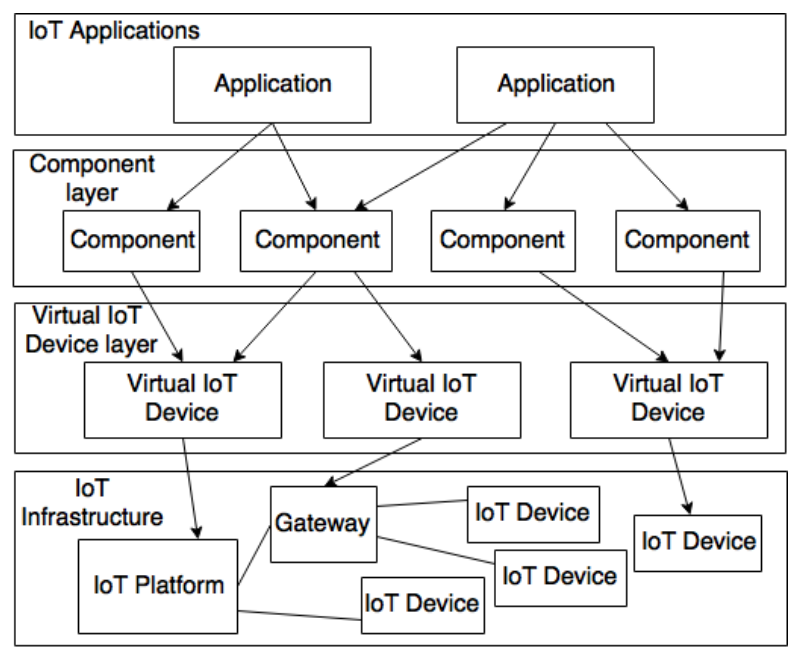

Figure 1. Software layers of the SMIoT architecture.

The IoT Infrastructure Layer represents the hardware and software that senses and actuates the physical world, and that stores historic values. This layer can consist of IoT devices, gateways and back-end IoT platforms. IoT devices are physical sensors and actuators. They can interact with the application directly (e.g. via Bluetooth), be mediated by a local gateway, or push their data into an IoT platform. The gateway can either be connected to the application over a local network or push the data to an IoT platform. The devices in this layer can be heterogeneous, and evolve over time. For instance, an IoT ecosystem can contain different types of temperature sensors, potentially relying on different communication technologies.

The Virtual IoT Device Layer consists of one or more virtual IoT devices. Each virtual IoT device defines one sensor or actuator in the IoT ecosystem. Multiple virtual IoT devices can point to the same infrastructural element. For instance, multiple sensors can be plugged on the same sensor kit. A type and technology is tied to each virtual IoT device. The type defines a sensor or actuator class. Examples are temperature sensor, lamp, lock... The technology defines the brand and model. Example lamp technologies are Philips Hue lightstrips/bulbs and Osram Lightify models. A uniform interface is assigned to each type and each technology of the same type must implement the uniform interface. The interface shields the upper layers from technological details. It also defines a uniform representation of the sensor's attributes. For example, a temperature value can be retrieved in ${ }^{\circ} \mathrm{C}$, ${ }^{\circ} \mathrm{K}$, or ${ }^{\circ} \mathrm{F}$. The virtual IoT device implementation transforms the values if necessary and passes a uniform representation to the upper layers. Application developers rely on those interfaces to invoke methods on IoT devices. If a sensor is replaced by another one provided by a different 
manufacturer, a new virtual IoT device implementation must be loaded.

The Component Layer models the application domain and consists of a set of components. Each component represents an asset in the application domain. For instance, rooms and patients can be components in a care environment. Similarly, trucks and trailers can be components in a fleet management ecosystem. Each component defines an intuitive interface to interact with the asset. Their implementation relies on the uniform interfaces provided by the virtual IoT device layer. A method in a component layer can invoke one or more methods in a virtual IoT device layer. For instance, retrieving the heartbeat of a patient component will simply result in the invocation of a heartbeat method in a heartbeat sensor. Similarly, to get the location of a truck, the location of a GPS sensor will be requested. In some circumstances, the mapping is less trivial. For example, consider a component that contains a heartbeat monitor. Other than a getHeartbeat () method, this component could also provide a method to developers that pushes a notification when a threshold heartbeat is exceeded. This requires intelligence at component level.

The Application Layer contains the applications in the IoT ecosystem. These applications invoke the interfaces provided by (a set of) components and interact with the physical environment. Hence, application developers do not require knowledge of IoT communication technologies or protocols.

\section{B. Software Components}

An overview of the different software components is shown in Figure 2. Apart from the previously discussed abstraction layers, software components for system administration and environment loading are shown. The software components for these tasks are further discussed below. Note that these software components rely on an Identity and Access Management (IAM) system to authenticate the users in the IoT ecosystem. Since IoT systems are typically integrated in an existing software infrastructure, often an existing IAM system can be used. Potentially new (types of) users need to be added. If not such system exists, a new IAM can be setup specifically for the users of IoT applications.

1) System Administration: The system administration module provides an interface to system administrators to create, read, update and delete three types of information. The first type is the information required to instantiate virtual IoT devices representing the sensors/actuators in the IoT ecosystem. This includes sensor type, communication parameters such as MAC or IP address and authentication credentials that (can be used to) grant access to the IoT device's functionality. Depending on the specific IoT device, adding, removing or updating this information requires going through a pairing procedure. The second type is the information required to instantiate the components in the IoT

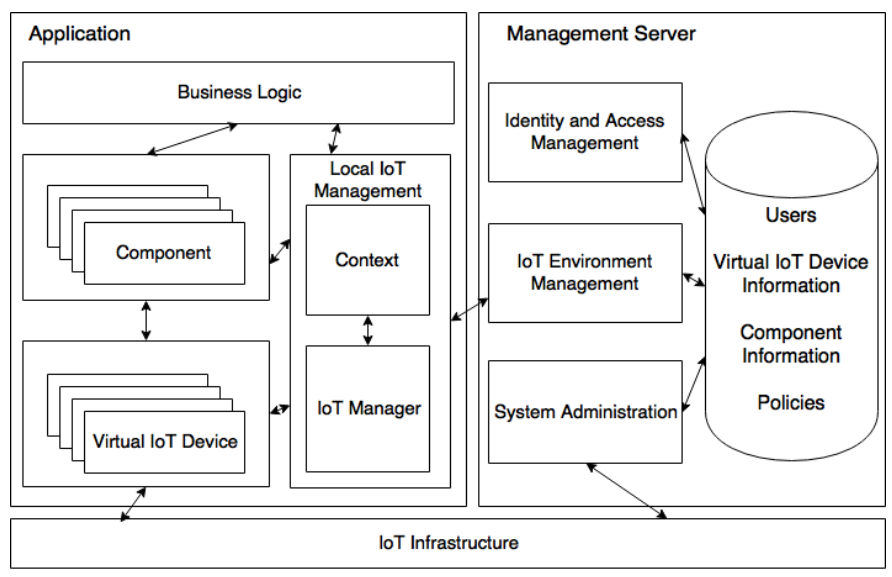

Figure 2. Software components of the SMIoT architecture.

ecosystem. Non-IoT related parameters are managed outside the SMIoT architecture, since this is typically information already managed by existing business processes (e.g. name and address of patients, information about the cargo in trucks). To instantiate the components, references to where this information can be obtained are stored. The IoT-related parameters constitute the set of virtual IoT devices linked to a specific asset and required for the IoT interfaces of the component. Long-term links are typically managed by the system administration module (e.g. the sensors and actuators installed in a care home). Short-term links can be dynamically created by business processes (e.g. when cargo is loaded in a truck, the sensors installed in the cargo hold of the truck are linked to the cargo). Finally, the third type are the policies used to determine which components are loaded in an application. The policy rules are defined using contextual parameters such as the identity of the user, location of the user and the type of application.

2) Environment Loading: The IoT environment (i.e. set of components and virtual IoT devices) loaded in an application depends on the context. The context Manager contained in the end-user application is responsible for collecting the necessary context parameters (e.g. identity of the user, location, type of application). When the context is collected, it is transferred to the IoT Environment Manager that uses the specified IoT policies to determine which components need to be loaded for the given context. Once the set of components is determined, the information to instantiate the components is collected. This includes the information about the linked virtual IoT devices and, potentially, links to other data sources managed by existing business processes. Once all the information is collected, it is returned to the application where the IoT Manager instantiates and initializes the received components and virtual IoT devices. 


\section{ANDROID FRAMEWORK}

This section presents a software framework 4 that supports developers with the implementation of the virtual IoT device layer. Although the framework presented in this section specifically targets Android, a similar approach can be taken for other platforms.

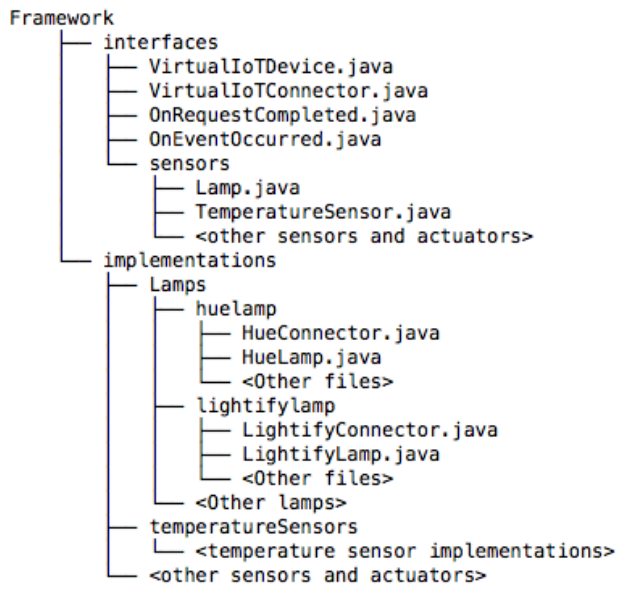

Figure 3. File hierarchy of the virtual IoT device framework.

Figure 3 provides an overview of the file hierarchy of the framework. The framework code is split in two folders: interfaces and implementations. The former contains generic framework code and defines the uniform interfaces to interact with sensors and actuators. The latter contains implementations of these interfaces for specific IoT technologies. The interfaces are defined as abstract methods in abstract classes. For each type of sensor/actuator an abstract subclass of VirtualIoTDevice is defined that specifies its interfaces. Two types of sensing interfaces can be distinguished: request-based and monitoring. Request-based calls are used when a sensor value is needed just once (e.g. requesting the temperature of a patient). Monitoring is used when a continuous stream of data is required (e.g. monitoring the heart rate of a patient). With request-based interfaces, the application expects a single response, while a call to a monitoring-type interface typically returns periodic responses. The interfaces are also defined as asynchronous. A callback parameter is added to each method declaration. This shields the component or application developer from the complexity of executing the interaction with IoT devices in a separate thread. This is required since interaction with IoT devices can introduce delays that are undesirable on the main thread. The framework defines two callback interfaces, one for monitoring and one for request-based sensing/actuating (see OnRequestCompleted Listing 1 below). An instance of this interface is passed as an parameter with each call to

${ }^{4}$ The source code of the Android framework can be found here: https://github.com/msec-kul/SMIoT_v1.git

\author{
Listing 1. OnRequestCompleted Callback Interface \\ public interface OnRequestCompleted $\langle\mathrm{T}>\{$ \\ public void onSuccess(T response); \\ \} \\ public void onfailure(Exception exception);
}

IoT devices by the application or component developers. These instances handle the response from the IoT device. The framework contains interface definitions for common sensors and actuators such as Lamp and TemperatureSensor.

Often several sensors/actuators are accessed via a single IoT Infrastructure element. For instance, multiple sensors can be plugged on a single embedded device or access to a set of sensors can be provided via a gateway or a cloud platform. To reduce code redundancy and resource use, the framework provides the abstract class VirtualIoTConnector. This is an internal software component of the virtual IoT device layer and, hence, not exposed to upper layers in the architecture. A virtual IoT connector represents a communication endpoint and provides an API used by virtual IoT devices to send messages to the infrastructural element from which its sensor data can be retrieved. These communication endpoints implement a part of the communication protocol (e.g. wrap data in a REST request) and the authentication protocol used by the infrastructural element. A virtual IoT connector is shared by the virtual IoT devices from which the data is available from the same infrastructural element. This is especially important for connection-oriented communication protocols for which it is undesirable/impossible to maintain multiple connections (e.g. Bluetooth).

Since the (type of) parameters required to initialize virtual IoT devices and connectors is typically different for each type of IoT device, both abstract classes contain a generic initialize method with Map $<$ String,Object $>$ as parameter. For each virtual IoT device and connector implementation a set of keys is defined by the developer that specify each parameter required to initialize the device or connector. For the connectors, these parameters will typically be related to the used communication and authentication technology (e.g. URL or Bluetooth MAC address). For the devices, these parameters will be related to the identification of the specific sensor available on the connector endpoint and, typically, also a virtual IoT connector instance. Based on the initialization interfaces, a generic device manager can automatically instantiate and initialize virtual IoT devices and connectors based on configuration files containing the required parameters.

Developers can add new types of sensors or actuators by creating a new abstract subclass of VirtualloTDevice and defining the interface for that specific sensor/actuator. Developers can add support for specific IoT devices by subclassing the abstract class(es) that define the uniform interfaces of the sensors/actuators available on the IoT device, defining the initialization parameters and implementing the initialization and uniform interface methods. Support 
for IoT devices containing one type of sensor/actuator is contained in one class. If an IoT device consists of multiple types of sensors/actuators, a subclass for each sensor/actuator is created, sharing a virtualiotConnector. The framework already contains support for several IoT devices. Examples are Hue lamps and Osram lamps. Since the framework defines asynchronous interfaces, the implementation needs to delegate the interaction with the IoT devices to a separate thread and trigger the callback when the operation is completed. To realize this, the implementations provided with the framework use the RXAndroid framework, which is an Android port of ReactiveX 5 . This framework gives finegrained control over the execution of operations on different threads. Since Android only allows UI operations on the main thread, our framework uses RXAndroid to execute the interactions with the IoT device on a worker thread while triggering the callback on the main thread. The obtained sensor values can then be show in the UI by the application developer, without overhead of inter-thread communication.

\section{Validation: a Fleet Management Ecosystem}

This section applies the architectural principles to a fleet management system. Three types of actors are distinguished in the fleet management system: truck drivers, logistics managers, and customers. Each actor uses an IoT-enabled application that supports the execution of their tasks. Truck drivers use an application that enables monitoring of the environmental parameters in their cabin and trailer. The application for the logistics managers provides an overview of the location of the different trucks, enabling them to respond to unexpected delays and, potentially, update the schedule for other trucks or notify the client. The customers have access to a track-and-trace application that allows them to monitor the state (i.e. environmental parameters) and location of their cargo items. This section first describes the application domain model after which two scenarios are discussed in more detail: the coupling and decoupling of trailers with tractor units, and cargo monitoring/track-andtrace.

\section{A. Application domain model}

Figure 4 provides a simplified class diagram of the mapping of the fleet management system to the component and virtual IoT device layers defined in the architecture. The IoT ecosystem consists of several types of assets that need to be monitored. These assets are represented by components in the IoT system. A truck consists of a tractor unit (TractorUnit) and a trailer (Trailer). The tractor unit's cabin contains IoT sensors to measure the temperature, air quality and location of the cabin. The trailer's loading space is monitored with temperature, pressure and humidity sensors. Some IoT interfaces on components can

\footnotetext{
http://reactivex.io
}
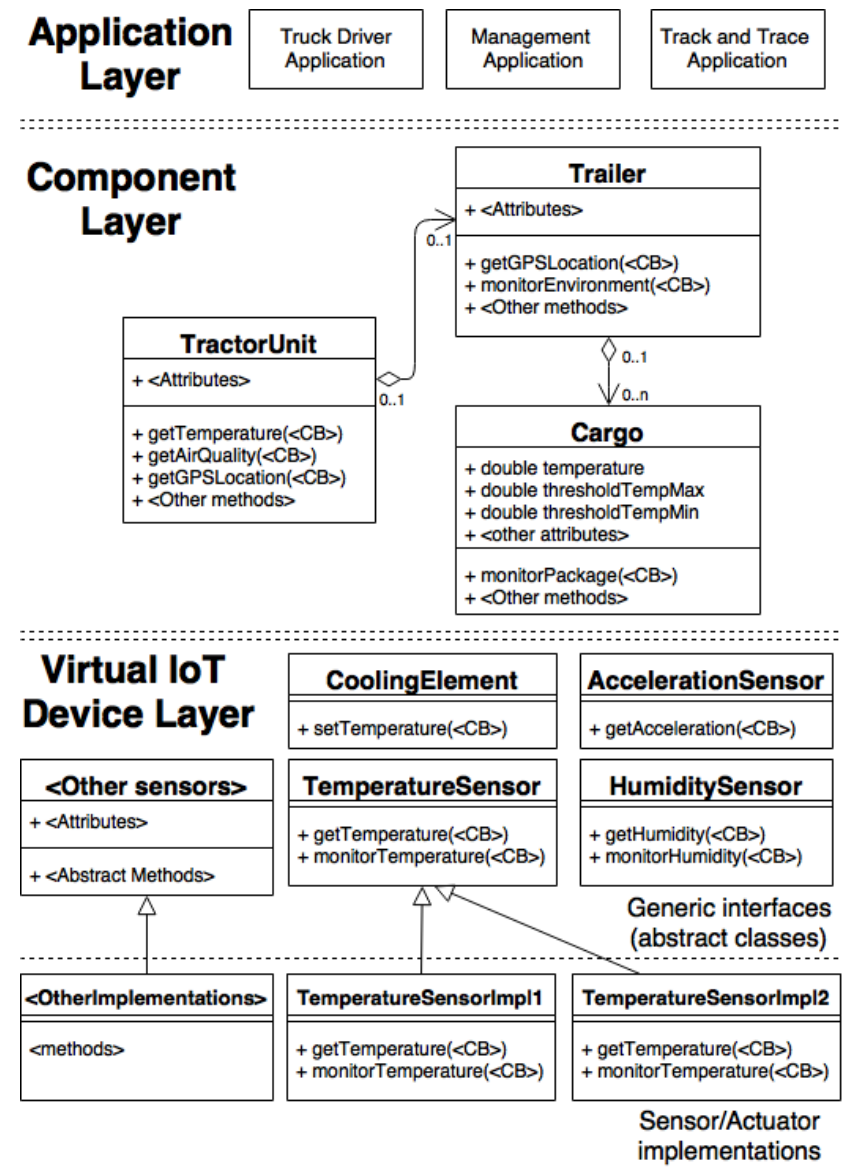

Figure 4. Simplified class diagram of the fleet management application domain model.

be mapped directly on methods on IoT devices (e.g. the getGPSLocation $(\langle C B\rangle))$. Other component interfaces are more complex and require interaction with several IoT devices and processing of the results. For instance, monitorPackage() of the Cargo component enables application developers to specify thresholds for specific environment parameters (i.e. temperature, pressure and humidity). When a measured parameter exceeds the set threshold (e.g. the temperature is too high) a notification is pushed to the application layer. When a specific Cargo item does not contain dedicated IoT equipment, the component layer can fall back to the sensors attached to the loading space of the TractorUnit.

\section{B. Scenario 1: Coupling and decoupling of a trailer and tractor unit}

Tractor units are coupled with numerous trailers during their lifetime. The truck driver's application needs to load the currently coupled trailer. Bluetooth beacons installed in each tractor unit and trailer enable the driver's application to load the appropriate components and associated IoT devices. Whenever a trailer is coupled or decoupled a context change occurs, triggering the driver's application to load/unload the necessary components/virtual IoT devices. 


\section{Scenario 2: Cargo monitoring and track-and-trace}

All three applications should be able to monitor and track the cargo. The sensor types that need to be coupled depend on the equipment in the trailer. The application receives the appropriate configuration of components and virtual IoT devices based on the identifier of the trailer and possibly other contextual parameters. Broken sensors can be replaced by more innovative sensors of the same type, potentially manufactured by a different vendor. The management server can receive IoT environment updates - typically initiated by operators -, and subsequently pass modifications to the IoT Manager modules. For some pieces of cargo, sensitive environment parameters such as temperature, pressure and humidity need to be controlled continuously. The type of parameters and their thresholds depend on the cargo. Therefore, some Cargo-components can contain their own set of sensors and actuators. When the context is loaded in an application, all required Cargo-components and their associated virtual IoT devices are loaded.

\section{DISCUSSION}

This section discusses the impact of more complex setups on the architecture and deliberates several aspects that need to be taken into account when using the architecture.

The system loads the IoT environment from a centralized platform based on the context. However, for some applications the context and, hence, the associated IoT environment rarely changes (e.g. a care application installed on a gateway of a care home). For these types of applications it can be beneficial to persist the IoT environment locally and provide support for offline loading of the environment. Further, some use cases require users to pair IoT devices on-the-fly with components. For instance, if a patient visits a medical center, a set of sensors are attached to the patient. These sensors need to be linked to the patient in the application of the caregiver. In these ad hoc situations, a mechanism for pairing locally without having to modify parameters in the cloud would increase the user friendliness of the system.

Several non-functional aspects related to IoT device selection (i.e. aspects other than which IoT device interfaces are supported) can have a significant impact on the behavior of the application. Hence, it is not always possible to replace an IoT device with any other device providing the same interfaces. Applications may require a specific Quality of Service (QoS) from IoT devices to ensure the desired behavior. This can be specified via QoS parameters such as measurement resolution, latency, sampling frequency, security, form factor and communication range. For example, IoT devices using LPWAN technologies typically have a longer latency compared to devices using more low-range communication technologies such as Bluetooth. Depending on the use case a device with a specific measurement resolution or latency can be selected. For instance, for temperature monitoring in smart city applications an IoT device with long-range communication, low measurement resolution and sampling frequency may be selected while these sensors would not be adequate for climate control applications. Further, some applications have advanced access control requirements with respect to IoT device interfaces. For instance, the owner of a house should always be able to open the smart lock of his front door, while caregivers should only be able to access the house when they have a scheduled appointment. Whether or not these advanced scenarios can be realized depends on the access control technology supported by the smart lock. Devices that support advanced access control technologies can be used in application domains with complex security requirements such as home care systems, while less complex devices can, for instance, be used for physical access control to a parking lot for employees.

The virtual IoT device framework makes abstraction of the monitoring frequency. The system administrator can specify the frequency at which sensor values are monitored. For IoT devices that support monitoring via, for instance, publishsubscribe messaging protocols such as MQTT this is typically defined as a parameter on the IoT device. For devices that do not support these types of protocols, the monitoring interface can be implemented via software-based polling by the virtual IoT device. Although this enables more flexibility in the specification of the monitoring frequency (i.e. the monitoring frequency can be provided as a parameter to the virtual IoT device), it can negatively impact the performance of the application (e.g. computational load, battery life). This is especially the case if sensor values should not be monitored at a fixed frequency but only if they pass a certain threshold (e.g. trigger an alarm if the temperature is too high). In these case, polling also significantly reduces the battery life of the sensor.

The virtual IoT device and component layers provide a high-level abstraction of the underlying IoT infrastructure to application developers. In some cases, even significant changes in the underlying infrastructure have no impact on the application layer. For instance, presence of people can be detected either via infrared sensors or via cameras. In some care homes, cameras may be used for presence detection because they are also used for security services. In other care homes, infrared sensor may be selected for cost reasons. The application developer can use the same presence interface on the component (e.g. Room), regardless of the underlying infrastructure. However, some changes in the underlying infrastructure will trigger changes in the component APIs provided to application developers. For instance, fall detection systems working with accelerometers will typically be used via the component Patient, while systems working with cameras will be used via, for instance, Room components.

Most applications contain both IoT and non-IoT related functionality. This is also reflected in the interfaces provided by the components to application developers. For 
example, fleet management applications typically need to have access to the contents of the Cargo. Hence, the IoTrelated interfaces of components are often complemented with interfaces supporting more traditional application-level operations. For systems that are retrofitted to include IoT applications, often components can wrap existing object representations of assets and add the IoT-related interfaces to the already existing functionality.

\section{CONCLUSION}

This paper presented the SMIoT architecture that provides guidelines for the building maintainable and flexible IoT ecosystems. The presented abstraction layers allow for smooth propagation of changes and updates to the ecosystem, and adaptation of the application when the user context changes. It further hides low-level IoT operations towards application developers. Hence, application developers can focus on implementing business logic and intuitive user interfaces without being an expert in IoT sensor technology. The architectural insights were incorporated in an Android framework and validated through the design and development of a fleet management ecosystem consisting of various IoT components.

\section{REFERENCES}

[1] A. Al-Fuqaha, M. Guizani, M. Mohammadi, M. Aledhari, and M. Ayyash, "Internet of things: A survey on enabling technologies, protocols, and applications," IEEE Communications Surveys Tutorials, vol. 17, pp. 2347-2376, Fourthquarter 2015.

[2] Z. M. Fadlullah, M. M. Fouda, N. Kato, A. Takeuchi, N. Iwasaki, and Y. Nozaki, "Toward intelligent machine-tomachine communications in smart grid," IEEE Communications Magazine, vol. 49, pp. 60-65, April 2011.

[3] R. Sanchez-Iborra and M.-D. Cano, "State of the art in lp-wan solutions for industrial iot services," Sensors, vol. 16, no. 5, 2016.

[4] L. Magnoni, "Modern messaging for distributed sytems," in Journal of Physics: Conference Series, vol. 608, p. 012038, IOP Publishing, 2015.

[5] J. Swetina, G. Lu, P. Jacobs, F. Ennesser, and J. Song, "Toward a standardized common $\mathrm{m} 2 \mathrm{~m}$ service layer platform: Introduction to onem2m," IEEE Wireless Communications, vol. 21, pp. 20-26, June 2014.

[6] R. Zgheib, E. Conchon, and R. Bastide, Engineering IoT Healthcare Applications: Towards a Semantic Data Driven Sustainable Architecture, pp. 407-418. Cham: Springer International Publishing, 2017.

[7] S. K. Datta, R. P. F. da Costa, C. Bonnet, and J. Hrri, "onem $2 \mathrm{~m}$ architecture based iot framework for mobile crowd sensing in smart cities," in 2016 European Conference on Networks and Communications (EuCNC), pp. 168-173, June 2016.

[8] S. Mumtaz, A. Alsohaily, Z. Pang, A. Rayes, K. F. Tsang, and J. Rodriguez, "Massive internet of things for industrial applications: Addressing wireless iiot connectivity challenges and ecosystem fragmentation," IEEE Industrial Electronics Magazine, vol. 11, pp. 28-33, March 2017.
[9] R. Lea and M. Blackstock, "City hub: A cloud-based iot platform for smart cities," in 2014 IEEE 6th International Conference on Cloud Computing Technology and Science, pp. 799-804, Dec 2014.

[10] H. Demirkan, "A smart healthcare systems framework," IT Professional, vol. 15, pp. 38-45, Sept 2013.

[11] F. Yang, N. Matthys, R. Bachiller, S. Michiels, W. Joosen, and D. Hughes, "Micropnp: Plug and play peripherals for the internet of things," in Proceedings of the Tenth European Conference on Computer Systems, EuroSys '15, (New York, NY, USA), pp. 25:1-25:14, ACM, 2015.

[12] M. Soliman, T. Abiodun, T. Hamouda, J. Zhou, and C. H. Lung, "Smart home: Integrating internet of things with web services and cloud computing," in 2013 IEEE 5th International Conference on Cloud Computing Technology and Science, pp. 317-320, Dec 2013.

[13] P. Desai, A. Sheth, and P. Anantharam, "Semantic gateway as a service architecture for iot interoperability," in 2015 IEEE International Conference on Mobile Services, pp. 313-319.

[14] R. Roman, J. Zhou, and J. Lopez, "On the features and challenges of security and privacy in distributed internet of things," Computer Networks, vol. 57, no. 10, pp. 2266 - 2279, 2013. Towards a Science of Cyber Security Security and Identity Architecture for the Future Internet.

[15] G. D. Abowd, A. K. Dey, P. J. Brown, N. Davies, M. Smith, and P. Steggles, "Towards a better understanding of context and context-awareness," in Proceedings of the 1st International Symposium on Handheld and Ubiquitous Computing, HUC '99, (London, UK, UK), pp. 304-307, Springer-Verlag.

[16] M. Baldauf, S. Dustdar, and F. Rosenberg, "A survey on context-aware systems," Int. J. Ad Hoc Ubiquitous Comput., vol. 2, pp. 263-277, June 2007.

[17] C. Perera, A. Zaslavsky, P. Christen, and D. Georgakopoulos, "Context aware computing for the internet of things: A survey," IEEE Communications Surveys Tutorials, vol. 16, pp. 414-454, First 2014.

[18] H. Xiao, Y. Zou, J. Ng, and L. Nigul, "An approach for context-aware service discovery and recommendation," in 2010 IEEE International Conference on Web Services, pp. 163-170, July 2010.

[19] A. K. Dey, "Understanding and using context," Personal Ubiquitous Comput., vol. 5, pp. 4-7, Jan. 2001.

[20] T. Gu, H. K. Pung, and D. Q. Zhang, "A serviceoriented middleware for building contextaware services," Journal of Network and Computer Applications, vol. 28, no. 1, pp. 1 $18,2005$.

[21] A. Rahmati and H. V. Madhyastha, "Context-specific access control: Conforming permissions with user expectations," in Proceedings of the 5th Annual ACM CCS Workshop on Security and Privacy in Smartphones and Mobile Devices, SPSM '15, (New York, NY, USA), pp. 75-80, ACM, 2015.

[22] A. Put and B. De Decker, "Pacco: Privacy-friendly access control with context," in Proceedings of the 13th International Joint Conference on e-Business and Telecommunications, ICETE 2016, (Portugal), pp. 159-170, SCITEPRESS - Science and Technology Publications, Lda, 2016.

[23] C. Doukas and F. Antonelli, "Compose: Building smart amp; context-aware mobile applications utilizing iot technologies," in Global Information Infrastructure Symposium - GIIS 2013, pp. 1-6, Oct 2013.

[24] D. Carlson and A. Schrader, "Dynamix: An open plug-andplay context framework for android," in 2012 3rd IEEE International Conference on the Internet of Things, pp. 151158, Oct 2012. 